\title{
Massive intraventricular haemorrhage from aneurysmal rupture: patient proportions and eligibility for intraventricular fibrinolysis
}

\author{
Dennis J. Nieuwkamp • Bon H. Verweij • \\ Gabriël J. E. Rinkel
}

Received: 8 May 2009/Revised: 7 August 2009/Accepted: 11 September 2009/Published online: 13 October 2009

(C) The Author(s) 2009. This article is published with open access at Springerlink.com

\begin{abstract}
Massive intraventricular haemorrhage (IVH) complicating aneurysmal subarachnoid haemorrhage (SAH) is associated with a poor prognosis. Small observational studies suggest favourable results from fibrinolysis of the intraventricular blood. We performed an observational study on IVH in a large series of patients with SAH to assess the proportion of patients that may benefit from fibrinolytic treatment. From our prospective database we retrieved patients with aneurysmal SAH admitted between January 2000 and January 2005. We calculated the proportion of patients with massive IVH and the proportion of patients that are eligible for fibrinolysis on basis of clinical and CT-scan characteristics and assessed neurological outcome in a treatment strategy without fibrinolysis. Poor neurological condition was defined as World Federation of Neurological Surgeons scale 4 and 5 , poor outcome as death or dependence 3 months after SAH. Of the 573 patients admitted with aneurysmal SAH, 59 (10\%; 95\% confidence interval CI 8-13\%) had massive IVH, of which 55 were in poor clinical condition. For these 55 patients, the case-fatality rate was $78 \%(95 \%$ CI $66-88 \%)$ and the proportion with poor outcome $91 \%$ (95\% CI $81-97 \%$ ). Of
\end{abstract}

D. J. Nieuwkamp ( $)$ · G. J. E. Rinkel

Department of Neurology, C03.236,

University Medical Centre Utrecht,

Rudolf Magnus Institute of Neuroscience,

P.O. Box 85500, 3584 CX Utrecht, The Netherlands

e-mail: d.nieuwkamp@umcutrecht.nl

B. H. Verweij

Department of Neurosurgery, C03.236,

University Medical Centre Utrecht,

Rudolf Magnus Institute of Neuroscience,

P.O. Box 85500, 3584 CX Utrecht, The Netherlands the 55 patients, $31(56 \%$, and $5 \%$ of all patients $\mathrm{SAH}$ within the study period) fulfilled our eligibility criteria and were considered suitable for intraventricular fibrinolysis. At 3 months, 30 of these 31 eligible patients $(97 \%$; 95\% CI 85-100\%) had a poor outcome. Massive IVH occurs in $10 \%$ of patients with aneurysmal SAH. Half of these patients may benefit from intraventricular fibrinolysis. Without fibrinolysis outcome is almost invariably poor in these patients.

Keywords Aneurysm - Subarachnoid · Intraventricular . Haemorrhage · Fibrinolysis

\section{Introduction}

Massive intraventricular haemorrhage (IVH) after aneurysmal subarachnoid haemorrhage ( $\mathrm{SAH})$ is associated with a poor prognosis, but there are few data on the proportion of patients with massive IVH [11]. Approximately $85 \%$ of patients die within a few days if no treatment is implemented. In patients treated with external ventricular drainage (EVD) the case fatality rate is still close to $65 \%$ [14]. Several small case series on fibrinolysis of the intraventricular blood suggest favourable results, but numbers of treated and reported patients remain small $[2,3,5,12$, $15,19,20]$. The proportion of patients with aneurysmal SAH that are eligible for intraventricular fibrinolysis and the potential health gain in this subset of patients are unknown. We reviewed the records and radiographs of patients with an aneurysmal SAH to study the proportion of patients with severe IVH, the proportion of patients that in our opinion are eligible for EVD and fibrinolysis and the outcome in these patients from a strategy without intraventricular fibrinolysis. 


\section{Methods}

Identification of patients

Data from all patients with SAH admitted to our institution are prospectively entered into a database. We retrieved records of patients with aneurysmal SAH and IVH admitted between January 2000 and January 2005. Patients were included if the IVH was massive on admission or after rebleeding. Massive IVH was defined as a score of 7 or higher on the Graeb scale [4]. This scale adds the individual scores (maximum 12) for the lateral ventricles (1, trace of blood or mild bleeding; 2, less than half of the ventricle filled with blood; 3 , more than half of the ventricle filled with blood; 4 , ventricle filled with blood and expanded) and the third and fourth ventricles (1, blood present, ventricle size normal; 2 , ventricle filled with blood and expanded).

\section{Data collection}

We extracted the following baseline data: age, sex, clinical condition assessed by means of the World Federation of Neurological Surgeons scale (WFNS) [1] score at admission and at time of rebleeding if the intraventricular extension occurred after rebleeding, CT findings (amount of intraventricular blood, intracerebral extension, bicaudate index (BCI) $[6,18]$, presence of midline shift, aneurysm location), clinical course during admission, and outcome (Glasgow Outcome Scale) [8] at 3 months after SAH.

All three authors independently graded the amount of intraventricular blood and assessed eligibility for EVD and intraventricular fibrinolysis. Within the subset of patients with massive IVH we considered patients eligible for EVD and fibrinolysis if (1) patients were in poor clinical condition (WFNS IV or V); (2) ventricles were enlarged; and (3) no ICH was present that contributed to the poor clinical condition, according to the judgment of the authors after reviewing the $\mathrm{CT}$ scan. In case of conflicting opinions a consensus meeting was held.

\section{Outcome}

Outcome status at 3 months after SAH had been assessed by means of the Glasgow Outcome Scale (GOS). For the present study we calculated the proportion of patients who died and those with poor outcome (dead or dependent, i.e. GOS 1-3).

Data analysis

Within the group of patients with an aneurysmal SAH we calculated the proportion of patients with massive IVH and the proportion of patients eligible for EVD and intraventricular fibrinolysis based on CT scan and clinical characteristics. Furthermore, we calculated the proportion of patients with poor outcome for the entire group of patients with massive IVH and for the subgroup of patients who in our opinion were eligible for EVD and fibrinolysis.

\section{Results}

We identified 573 patients admitted with aneurysmal SAH, 59 (10\%; 95\% CI 8-13\%) had massive IVH (Fig. 1). The ages of the 59 patients ranged between 36 and 85 years with a mean age of 57 years; 39 patients $(66 \%)$ were women. Of these 59 patients 16 had initially been admitted with no or mild IVH, but developed massive IVH after rebleeding. Seventeen patients $(29 \%)$ had one or more episodes of rebleeding during the following clinical course.

Four of the 59 patients (7\%) had been admitted in a good neurological condition and remained in good neurological condition during admission. The median Graebscore for these four patients was 7 . They were independent at 3 months after SAH. The other 55 patients with massive

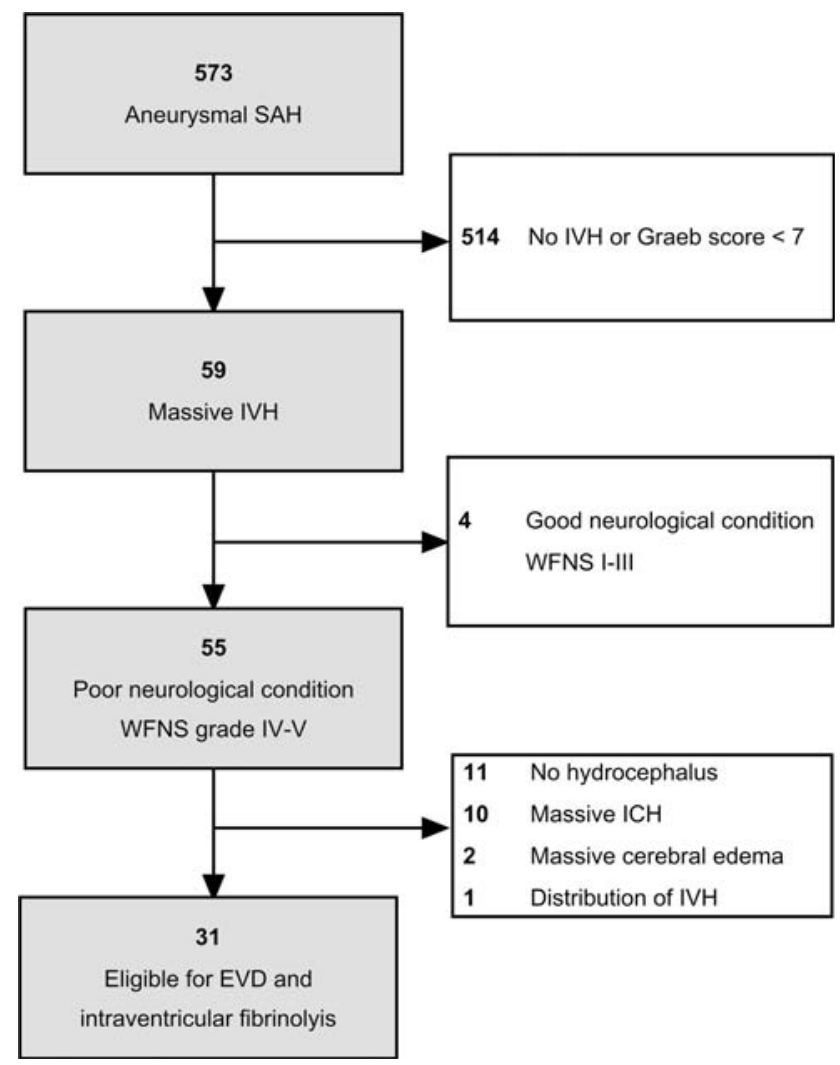

Fig. 1 Flowchart of SAH patients. SAH subarachnoid haemorrhage, $I V H$ intraventricular haemorrhage, WFNS World Federation of Neurological Surgeons scale, ICH intracerebral haemorrhage, EVD external ventricular drainage 
IVH were in poor clinical condition (Table 1). The median Graeb-score was 9 . Of the 59 patients, 25 did not fulfil our predefined eligibility criteria (good neurological condition in four patients, accompanying ICH contributing to the poor clinical condition in ten patients and no hydrocephalus in 11 patients). Three patients who fulfilled the criteria were considered ineligible nonetheless. Two of these patients were deemed to have a poor outcome regardless of treatment because of diffuse brain swelling with effacement of the basal cisterns. In the third patient the distribution of the IVH made the patient ineligible because the third and fourth ventricle, as well as the occipital horns of the lateral ventricles, were filled with blood, with no blood in the frontal horns so that EVD without fibrinolysis would be the initial treatment of choice in our opinion. 31 out of the 59 patients with massive IVH (53\% of all patients with severe IVH; $5 \%$ of all patients admitted with aneurysmal SAH within the study period) fulfilled our predefined eligibility criteria and were considered suitable for EVD and intraventricular fibrinolysis.

CT scans demonstrated ICH in 28 patients (51\%). These were located in the frontal lobe in 20 patients, frontotemporally in two and in the temporal lobe in six patients. The mean volume of the accompanying ICH in the patients classified as eligible was $7.5 \mathrm{~cm}^{3}$ (SD 5.7) compared to $54.6 \mathrm{~cm}^{3}$ (SD 23.6) in the patients classified as not eligible for intraventricular fibrinolysis.

After 3 months, 50 out of the 55 patients $(91 \%)$ had a poor outcome. In 17 patients $(31 \%)$ the aneurysm was treated, in nine patients by means of neurosurgical clipping and in eight patients by means of endovascular coiling. Of the 31 patients that would have been eligible for EVD and intraventricular fibrinolysis, 13 patients (42\%) had been treated with EVD only. After 3 months, 24 of the 31 patients (77\%) had died and $6(19 \%)$ were left dependent. Only one patient recovered to an independent state, this patient had a WFNS score of IV and was treated with EVD. Thus, 30 of the 31 patients (97\%) considered eligible for EVD and intraventricular fibrinolysis had poor outcomes.

\section{Discussion}

In our cohort of patients, massive IVH occurred in $10 \%$ of patients with aneurysmal SAH. Half of these patients would have been eligible for EVD and intraventricular fibrinolysis according to our criteria on the basis of neurological condition and CT-scan characteristics. All but one of the eligible patients (97\%) had a poor outcome after a strategy without intraventricular fibrinolysis.

Four patients with severe IVH remained in a good neurological condition during admission and were independent of help for activities of daily living 3 months after the SAH. The good outcome in these patients confirms our opinion that these patients should not receive intraventricular fibrinolysis and should be excluded in future clinical trials on intraventricular fibrinolysis after aneurysmal SAH.

The proportion of one out of three patients with rebleeding is higher than in series of SAH patients in general. Possible explanations for this high proportion include a longer exposure time because of postponement of treatment of the ruptured aneurysm in these very poor-grade patients. Furthermore, one-third of the patients in this series developed massive IVH after a proven rebleeding, and in the remaining patients out of hospital rebleeding may have occurred in a considerable proportion, because massive IVH is found more often in patients with rebleeding than in patients with a single bleed [9]. Thus, a large proportion of the patients in our series had already had an episode of rebleeding at study entry. Since a first episode of rebleeding is a risk factor for further episodes, this is

Table 1 Outcome and treatment characteristics of patients in poor neurological condition (WFNS IV or V)

\begin{tabular}{|c|c|c|c|c|c|c|}
\hline Patients & $\begin{array}{l}\text { Death No. } \\
\text { of patients }\end{array}$ & Percent & $(95 \% \mathrm{CI})$ & $\begin{array}{l}\text { Death or dependent } \\
\text { No. of patients }\end{array}$ & Percent & $(95 \% \mathrm{CI})$ \\
\hline \multicolumn{7}{|l|}{ All patients } \\
\hline$n=55$ & 43 & 78 & $(66-88)$ & 50 & 91 & $(81-97)$ \\
\hline \multicolumn{7}{|c|}{ Patients eligible for intraventricular fibrinolysis } \\
\hline$n=31$ & 24 & 77 & $(60-90)$ & 30 & 97 & $(85-100)$ \\
\hline $\mathrm{EVD}+(n=13)$ & 6 & 46 & $(21-73)$ & 12 & 92 & $(68-100)$ \\
\hline $\mathrm{EVD}-(n=18)$ & 18 & 100 & $(85-100)$ & 18 & 100 & $(85-100)$ \\
\hline \multicolumn{7}{|c|}{ Patients not eligible for intraventricular fibrinolysis } \\
\hline$n=24$ & 19 & 79 & $(60-92)$ & 20 & 83 & $(65-94)$ \\
\hline $\mathrm{EVD}+(n=7)$ & 3 & 43 & $(12-78)$ & 4 & 57 & $(22-88)$ \\
\hline $\mathrm{EVD}-(n=17)$ & 16 & 94 & $(74-100)$ & 16 & 94 & $(74-100)$ \\
\hline
\end{tabular}

WFNS World Federation of Neurological Surgeons scale, EVD external ventricular drainage 
another explanation for the high proportion of patients with rebleeding in our study [7].

The proportion of patients with massive IVH we found is comparable with the $11 \%$ that was found before in a cohort of 219 SAH patients [11]. Our results regarding outcome are in concordance with the results found in a systemic review in which $79 \%$ of patients with massive IVH from aneurysmal SAH died and 93\% had a poor outcome, regardless of treatment with EVD or conservative treatment. In this review, the reduction in case fatality from 84 to $67 \%$ for patients treated with EVD compared with conservative treatment was not statistically significant and the poor outcome rates were similar (93\%) for both treatment strategies [14]. In our series we cannot compare treatment with EVD with conservative treatment because of selection bias. Treatment was not according to a pre-specified protocol, but left at the discretion of the treating physician or surgeon. Treatment with EVD has probably been withheld in some patients because of expected drain obstruction and poor outcome, which may explain the small proportion of patients treated with EVD. Withdrawal of care could have contributed to the poor outcome rates. Especially in these poor-grade patients, one should beware the possibility of increasing poor outcome rates as a result of self-fulfilling prophecy. Another limitation of our study is that the assessment whether or not the accompanying ICH was a major contributor to poor clinical condition was somewhat arbitrary. Even so, we feel that in patients with a small accompanying ICH that does not importantly contribute to poor clinical condition, its presence should not be an absolute contraindication for intraventricular fibrinolysis. EVD and intraventricular fibrinolysis in primary ICH accelerates the resolution of intraventricular blood clots and seems to do so with a safety profile similar to that of EVD without fibrinolysis [13].

An advantage of our study is that it describes a large series of homogeneous patients. Most other articles describe small numbers of patients with different underlying causes for the haemorrhage. The underlying cause is of great importance for the prognosis $[10,16]$. The amount of IVH is a risk factor for poor outcome in patients with IVH $[11,16,17]$. Because we dichotomised the amount of blood we could not properly examine the exact relation between the amount of blood and outcome. In addition, the current study is the first that describes outcome in the subset of patients that would have been eligible for intraventricular fibrinolysis.

In conclusion, massive IVH is an important problem in SAH. It occurs in $10 \%$ of patients, and half of these are eligible for intraventricular fibrinolysis. Without fibrinolysis, outcome is almost invariably poor. With fibrinolysis it is unknown, although evidence that comes from small case series suggests a clear benefit. We must await results from the CLEAR-IVH trial (Clot Lysis: Evaluating Accelerated Resolution of Intraventricular Haemorrhage) to clarify the effectiveness of intraventricular fibrinolysis in IVH after ICH. Likewise, an RCT that assesses intraventricular fibrinolysis in poor-grade aneurysmal SAH patients is clearly warranted, considering the very high poor outcome rates in these patients, even when treated with EVD.

Acknowledgment This study was funded in part by a grant from the Netherlands Brain Foundation to Dr. B.H. Verweij.

Conflict of interest statement The authors declare no conflict of interest.

Open Access This article is distributed under the terms of the Creative Commons Attribution Noncommercial License which permits any noncommercial use, distribution, and reproduction in any medium, provided the original author(s) and source are credited.

\section{References}

1. Drake CG (1988) Report of World-Federation-of-NeurologicalSurgeons Committee on a universal subarachnoid hemorrhage grading scale. J Neurosurg 68:985-986

2. Findlay JM, Grace MG, Weir BK (1993) Treatment of intraventricular hemorrhage with tissue plasminogen activator. Neurosurgery 32:941-947

3. Findlay JM, Jacka MJ (2004) Cohort study of intraventricular thrombolysis with recombinant tissue plasminogen activator for aneurysmal intraventricular hemorrhage. Neurosurgery 55:532537

4. Graeb DA, Robertson WD, Lapointe JS, Nugent RA, Harrison PB (1982) Computed tomographic diagnosis of intraventricular hemorrhage. Etiology and prognosis. Radiology 143:91-96

5. Hall B, Parker D Jr, Carhuapoma JR (2005) Thrombolysis for intraventricular hemorrhage after endovascular aneurysmal coiling. Neurocrit Care 3:153-156

6. Hasan D, Vermeulen M, Wijdicks EF, Hijdra A, van Gijn J (1989) Management problems in acute hydrocephalus after subarachnoid hemorrhage. Stroke 20:747-753

7. Hijdra A, Vermeulen M, van Gijn J, van Crevel H (1987) Rerupture of intracranial aneurysms: a clinicoanatomic study. J Neurosurg 67:29-33

8. Jennett B, Bond M (1975) Assessment of outcome after severe brain damage. Lancet 1(7905):480-484

9. Linn FH, Rinkel GJ, Algra A, van Gijn J (2000) The notion of "warning leaks" in subarachnoid haemorrhage: are such patients in fact admitted with a rebleed? J Neurol Neurosurg Psychiatry 68:332-336

10. Marti-Fabregas J, Piles S, Guardia E, Marti-Vilalta JL (1999) Spontaneous primary intraventricular hemorrhage: clinical data, etiology and outcome. J Neurol 246:287-291

11. Mayfrank L, Hutter BO, Kohorst Y, Kreitschmann-Andermahr I, Rohde V, Thron A, Gilsbach JM (2001) Influence of intraventricular hemorrhage on outcome after rupture of intracranial aneurysm. Neurosurg Rev 24:185-191

12. Mayfrank L, Rohde V, Gilsbach JM (1999) Fibrinolytic treatment of intraventricular haemorrhage preceding surgical repair of ruptured aneurysms and arteriovenous malformations. $\mathrm{Br} \mathrm{J}$ Neurosurg 13:128-131 
13. Naff NJ, Hanley DF, Keyl PM, Tuhrim S, Kraut M, Bederson J, Bullock R, Mayer SA, Schmutzhard E (2004) Intraventricular thrombolysis speeds blood clot resolution: results of a pilot, prospective, randomized, double-blind, controlled trial. Neurosurgery $54: 577-583$

14. Nieuwkamp DJ, de Gans K, Rinkel GJ, Algra A (2000) Treatment and outcome of severe intraventricular extension in patients with subarachnoid or intracerebral hemorrhage: a systematic review of the literature. J Neurol 247:117-121

15. Rohde V, Schaller C, Hassler WE (1995) Intraventricular recombinant tissue plasminogen activator for lysis of intraventricular haemorrhage. J Neurol Neurosurg Psychiatry 58:447-451

16. Roos YB, Hasan D, Vermeulen M (1995) Outcome in patients with large intraventricular haemorrhages: a volumetric study. J Neurol Neurosurg Psychiatry 58:622-624

17. Tuhrim S, Horowitz DR, Sacher M, Godbold JH (1999) Volume of ventricular blood is an important determinant of outcome in supratentorial intracerebral hemorrhage. Crit Care Med 27:617621

18. van Gijn J, Hijdra A, Wijdicks EF, Vermeulen M, van Crevel H (1985) Acute hydrocephalus after aneurysmal subarachnoid hemorrhage. J Neurosurg 63:355-362

19. Varelas PN, Rickert KL, Cusick J, Hacein-Bey L, Sinson G, Torbey M, Spanaki M, Gennarelli TA (2005) Intraventricular hemorrhage after aneurysmal subarachnoid hemorrhage: pilot study of treatment with intraventricular tissue plasminogen activator. Neurosurgery 56:205-213

20. Vereecken KK, van Havenbergh T, de Beuckelaar W, Parizel PM, Jorens PG (2006) Treatment of intraventricular hemorrhage with intraventricular administration of recombinant tissue plasminogen activator. A clinical study of 18 cases. Clin Neurol Neurosurg 108:451-455 